\title{
Genre et mathématiques dans les images des manuels scolaires en France
}

\section{Carole Brugeilles et Sylvie Cromer}

\section{(2) OpenEdition}

\section{Journals}

Édition électronique

URL : http://journals.openedition.org/trema/2599

DOI : $10.4000 /$ trema.2599

ISSN : 2107-0997

Éditeur

Faculté d'Éducation de l'université de Montpellier

Édition imprimée

Date de publication : 1 décembre 2011

Pagination : 142 - 154

ISSN : 1167-315X

Référence électronique

Carole Brugeilles et Sylvie Cromer, «Genre et mathématiques dans les images des manuels scolaires en France », Tréma [En ligne], 35 - 36 | 2011, mis en ligne le 01 décembre 2013, consulté le 01 mai 2019. URL : http://journals.openedition.org/trema/2599; DOI : 10.4000/trema.2599

Ce document a été généré automatiquement le 1 mai 2019.

Trema 


\title{
Genre et mathématiques dans les images des manuels scolaires en France
}

\author{
Carole Brugeilles et Sylvie Cromer
}

1 Bien que le principe d'égalité entre les sexes se soit, en Europe, au fil des siècles consolidé en prenant appui sur les textes internationaux et l'acquis communautaire, les inégalités entre les sexes persistent, y compris dans les systèmes éducatifs où la scolarisation des filles et des garçons est paritaire $^{1}$ (cf. notamment BAUDINO, 2007 ; JARLÉGAN, 2009 ; réseau Eurydice 2010). Un double constat est unanimement établi dans les pays de l'OCDE (Organisation de coopération et de développement économiques): « une suprématie des filles doublée partout d'une orientation plus favorable des garçons qui parviennent aux meilleurs niveaux » (BAUDELOT, ESTABLET, 2007, p.91). Comment expliquer la réussite paradoxale des garçons, monopolisant les

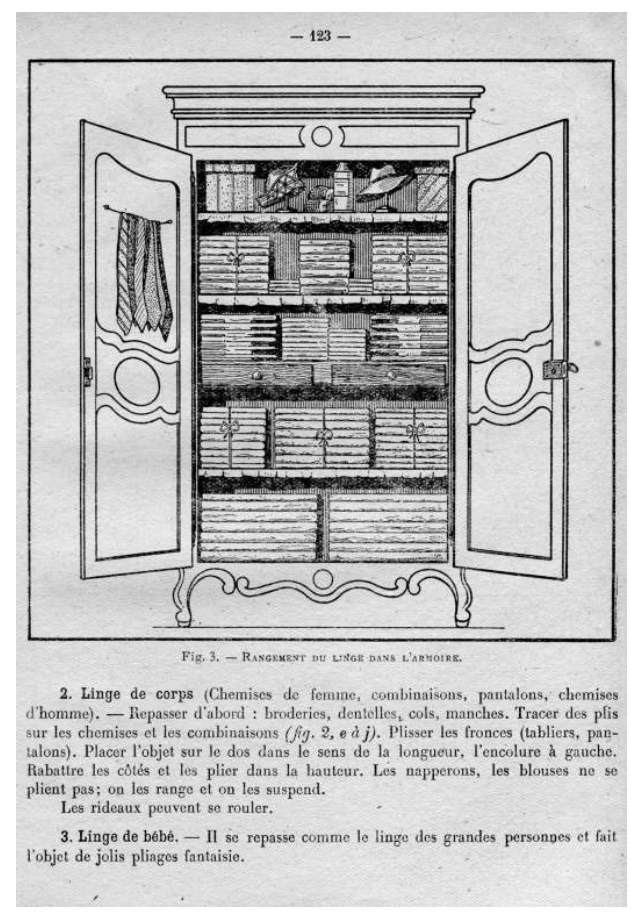
filières de prestige offrant davantage de débouchés, tout en connaissant davantage de difficultés scolaires, et l'échec paradoxal des filles, accomplissant mieux leur métier d'élève mais restreignant leur choix d'orientation? Les stéréotypes de sexe prégnants au travers de pratiques, de discours, de représentations, qui sont aussi relayés par l'école, constituent une réponse partielle. 
2 Les représentations sexuées et sexistes véhiculées par les manuels scolaires et autres matériels didactiques peuvent apparaître comme une question mineure, au regard d'autres obstacles à l'égale scolarisation des filles identifiés. De plus, la question ne seraitelle pas dépassée, étant donné les nombreuses études réalisées depuis des décennies à l'échelle mondiale, dont les résultats sont, rappelons-le, étonnamment convergents? Pourtant, selon la démonstration de Rae LESSER BLUMBERG (2007), les stéréotypes s'avèrent l'invisible pierre d'achoppement sur le chemin de l'égalité: "To return to a dry land metaphor, it is time to strip the camouflage off the hidden obstacle of gender bias in learning materials. Only then can it be seen for what it is, analyzed and, hopefully, pushed off the road. » (p.6). En effet, ceux-ci persistent, quelle que soit la performance du système éducatif, et sont d'ailleurs régulièrement pointés du doigt dans les rapports de l'Education pour tous (par exemple Unesco, 2003-2004). Le récent état des lieux du réseau Eurydice conclut pour sa part sans ambiguité : «Les manuels scolaires montrent des images stéréotypées des hommes et des femmes et peu d'entre eux s'attachent à aller à l'encontre des représentations stéréotypées des hommes et des femmes et à faire un rééquilibrage (...)». (2010, p.75). La comparaison d'études diachroniques, comme le fait BLUMBERG retraçant la saga de la lutte contre les préjugés sexistes aux États-Unis (2007, notamment p. 12-19), montre de faibles améliorations en 35 ans de lutte, alors même que manuels et autres matériels didactiques restent très utilisés par les enseignant-e-s et les élèves et qu'ils reflètent les curricula.

3 C'est pourquoi nous avons choisi de remettre à l'ordre du jour l'analyse des manuels scolaires avec la création en 2003 du Réseau international de recherche sur les représentations sexuées (RIRRS). Dans la lignée de travaux menés sur les représentations sexuées, initiés en 1997 au sein de l'association «Du côté des filles » avec le programme de recherche européen "Attention Album ", notre démarche ${ }^{2}$ s'adosse aux concepts de genre et de représentation. Nous entendons par genre le rapport social qui divise l'humanité en deux groupes de sexe inégaux hiérarchisés et empruntons à Denise JODELET la définition de représentation: «forme de connaissance socialement élaborée et partagée, ayant une visée pratique et concourant à la construction d'une réalité commune à un ensemble social» $(1989$, p.53). L'originalité consiste à mettre en œuvre une méthode quantitative permettant l'étude de vastes corpus et d'établir, quand cela est possible, des comparaisons. Concrètement, il s'agit d'une part de recenser tous les personnages sans sélection a priori ; d'autre part de recueillir, grâce à un questionnaire, les différentes caractéristiques du " sexe social » du personnage, c'est-à-dire les éléments de son identité et de sa place socialement construites en fonction de son sexe et de son âge. Ces indices sont complexes et contingents, se médiatisent par le texte, l'image, la langue, ainsi que par la structure et l'usage mêmes du support. Autrement dit, non seulement filles, garçons, femmes, hommes sont décomptés, mais ils et elles sont recensés, du point de vue de leurs qualités, attributs, actions, activités, etc. ainsi que par leurs relations. La combinaison des indices permet d'esquisser le système de genre légitimé, c'est-à-dire de mettre au jour les identités et les rôles sociaux sexués définissant culturellement le masculin et le féminin. Ainsi saisir les représentations sexuées consiste à tenter de savoir ce qui, dans ces objets, à la fois productions culturelles et outils d'apprentissage, est donné à voir de la mise en ordre de la réalité du point de vue de l'organisation des sexes.

4 Notre choix s'est porté sur les manuels de mathématiques qui restent peu examinés, l'attention se focalisant plus souvent sur les ouvrages d'histoire, d'éducation civique ou de littérature (BOISSEAU et al., 2005, BRUILLARD, 2005 ; TISSERANT et WAGNER, 2008). Pourtant 
très peu de professeur-e-s des écoles sont issu-e-s de filières scientifiques (MICHEL, 2005, p.5) et ils recourent donc de manière importante aux manuels de mathématiques qui restent un outil d'apprentissage fortement utilisé dans les écoles en France (DURPAIRE, 2006, p.56). Du fait de la liberté pédagogique, les collections peuvent d'un cycle ou d'une classe à l'autre changer, plusieurs manuels à la fois peuvent servir dans une classe, on peut faire appel en plus à des photocopies. Il existe plus d'une vingtaine de collections de mathématiques pour l'école primaire, pour lesquelles les données sur la diffusion ne sont pas rendues publiques.

5 Dans cet article, nous considérons les personnages véhiculés par les images de trois collections entières de manuels de mathématiques parmi les plus répandues en France, d'après nos recoupements d'informations auprès de maisons d'édition, soit 15 ouvrages (voir bibliographie). Sont analysés les éléments suivants du sexe social : le sexe, l'âge, la fonction pédagogique, les activités ou actions, les attributs. Après un recensement de la population des images, nous confronterons le monde de l'enfance et le monde de l'âge adulte.

\section{Une nouvelle donne dans la classique hégémonie enfantine et masculine}

6 Dans les images des manuels, les personnages sont très nombreux. Dans les 15 manuels des trois collections, 3610 personnages au sexe et à l'âge identifiés sont dénombrés, soit une moyenne de 241 personnages par manuel. Les variations sont fortes selon les maisons d'édition : on a recensé 947 personnages dans la collection B, 1013 dans la collection A et 1650 dans la collection C. L'utilisation du personnage, qui reste massive, relève pourtant bien d'un choix éditorial et non d'une nécessité pédagogique.

7 Dans nombre de publications pour la jeunesse, les illustrations sont agrémentées préférentiellement de personnages d'enfants. Les manuels de mathématiques ne dérogent pas à la règle. Globalement, les enfants représentent $79 \%$ des personnages illustrés, soit près de huit personnages sur dix. On constate également la dominance numérique des personnages de sexe masculin observée dans d'autres corpus et ce, dans des proportions similaires, puisque $61 \%$ des personnages sont de sexe masculin. Ces prédominances « classiques » connaissent d'importantes variations selon les maisons d'édition, puisque la part des enfants varie entre $73 \%$ et $89 \%$ et celle des masculins entre $54 \%$ et $66 \%$. Mais la liberté éditoriale mentionnée plus haut ne conduit jamais à un renversement de ces tendances: le manuel reste le monde de l'enfance et du masculin. Si cette double hégémonie masculine et enfantine corrobore les constats statistiques faits dans les collections africaines de manuels scolaires (en Côte d'Ivoire, Cameroun, Togo par exemple, voir BRUGEILLES, CROMER, 2006, 2009), l'analyse des personnages selon leur sexe et leur âge révèle une nouvelle donne concernant leur classement.

Quelle que soit la collection, la hiérarchisation entre les quatre catégories de personnages est inchangée (graphique 1). La prééminence du garçon acquise dans chacune des collections et la marginalité de la femme, toujours en quatrième position, ne se démentent jamais. En revanche la visibilité des filles et des hommes - respectivement aux deuxième et troisième rangs - fluctue, au point que dans deux collections sur trois on s'approche de la parité filles/garçons (moins de deux points d'écart pour la collection B, quatre points pour la collection A). Ainsi le corpus français des images des manuels se 
distingue des corpus africains par deux résultats qui méritent d'être soulignés et interrogés. D'une part on peut approcher une quasi parité de sexe chez les personnages enfants ; d'autre part les adultes, même si leur nombre croît - irrégulièrement d'ailleurs au fil du cursus restent minoritaires. Or la comparaison de plusieurs corpus africains avait montré qu'au cours des cycles du primaire, l'importance des enfants cédait du terrain et que le poids du masculin se renforçait, le garçon s'effaçant devant l'homme, à la suprématie assurée. Comme si au fur et à mesure que des élèves - et tout particulièrement les filles - quittaient le système scolaire, il s'agissait de les préparer à cette entrée dans le monde adulte (et ainsi de la justifier ?). Quant aux élèves français scolarisés obligatoirement jusqu'à 16 ans, ils continuent de voir, en primaire tout du moins, dans les images essentiellement des personnages d'enfants, de surcroît presque autant de filles que de garçons. Notons toutefois qu'à certains niveaux de certaines collections, la présence des hommes la disputent à celle des enfants : au CE2 dans la collection A ; au CM1 dans la collection C. En revanche, dans l'enseignement secondaire, en l'occurrence dans les images des six manuels scientifiques de collège de $6 \mathrm{e}$ et $5 \mathrm{e}$ (trois de Mathématiques de 6e et trois de Physique-Chimie de $5 \mathrm{e}$ utilisés actuellement dans trois collèges de Toulouse), Capucine POMART (2009) établit une dominance masculine à $59 \%$ et le renversement de la dominance d'âge puisque les adultes représentent $72 \%$ des personnages.

L'accès à la parité traduit-il, suite aux différentes études menées, une sensibilisation au sexisme, étant donné les dates récentes de publication de nos manuels? Faute de pouvoir répondre à la question du pourquoi, nous chercherons à savoir comment est favorisée cette promotion des filles et le maintien de la présence des garçons tout au long de la scolarité primaire : qui sont ces personnages ? Comment et à quelle fin sont-ils utilisés?

Graphique 1 : Répartition des personnages selon leur sexe et leur âge par collection (en \%)

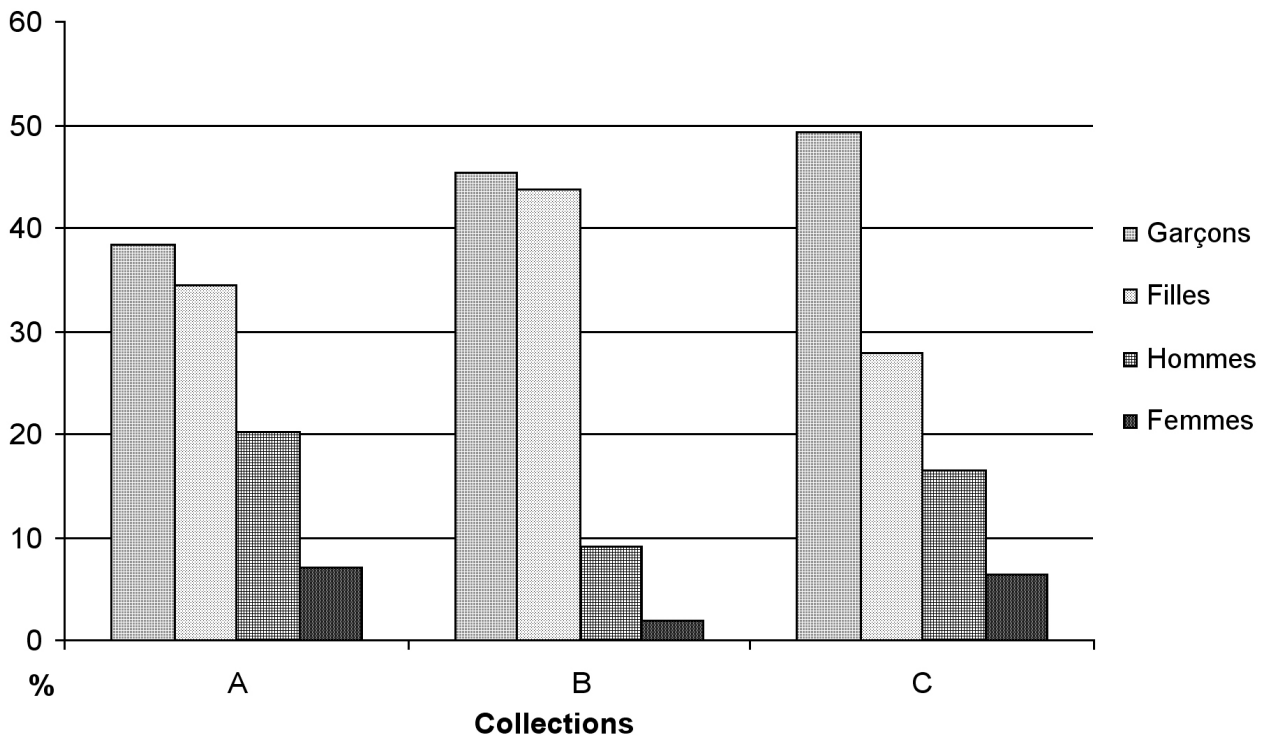

Source : Genre et manuels scolaires en France 


\section{Une parité sous conditions dans l'enfance}

9

Globalement, la population largement majoritaire des enfants (79\% des personnages) se compose de 1630 garçons et de 1222 filles, soit $57 \%$ de garçons et $43 \%$ de filles. Dans les manuels sont mobilisées deux grandes catégories de personnage: des personnages récurrents "conducteurs", uniquement des enfants dans les collections examinées, et des personnages « occasionnels » (enfants et adultes). Les premiers, signalés dès le début de l'ouvrage, accompagnent les élèves au fil des pages et au long de l'année scolaire. Les seconds apparaissent à quelques reprises seulement, voire une seule fois. Personnages occasionnels comme personnages conducteurs peuvent endosser de surcroît dans les images la fonction de "substitut ». Le substitut, de l'élève ou du maître, donne des consignes, des conseils, encourage, compatit... et se reconnaît par son interaction de nature scolaire avec l'usager-e du manuel. Conducteurs et substituts donnent de l'épaisseur au personnage, par l'identité étayée et affermie tout au long du livre, et/ou par l'interdépendance avec le lecteur ou la lectrice. On peut imaginer que ces personnages qui sortent de l'ordinaire retiennent davantage non seulement l'attention de l'élève, mais aussi la vigilance de la maison d'édition.

Or ces personnages mis en relief sont nombreux. $57 \%$ des personnages sont des personnages conducteurs dans l'ensemble des collections, avec un pourcentage variant entre $38 \%$ et $86 \%$ selon les collections. Un seul manuel sur quinze ne comporte aucun personnage conducteur (Pour comprendre les maths, CE2, 2004). On comprend ainsi la forte présence des enfants. De surcroit, les maisons d'édition ont le souci, dans leur grande majorité, de mettre en œuvre la parité chez ce type de personnage, assurant de cette manière la sortie de l'ombre des filles. Dans dix manuels sur quinze (tous les manuels de J'apprends les maths ; Cap maths du CP au CE2 ; Pour comprendre les maths du CP au CE1), on rencontre un couple mixte de personnages conducteurs et dans deux manuels, deux conductrices et deux conducteurs (Cap maths CM1 et CM2 de 2008). Deux manuels d'une même collection (Pour comprendre les maths, CM1 et CM2 de 2007 et 2005) choisissent un seul conducteur masculin: personnage de couleur verte, il est non humain, mais son prénom Mathéo, sa tenue vestimentaire et ses actions l'humanisent en personnage de sexe masculin. La vedette n'est donc pas donnée à un petit garçon proprement dit, mais néanmoins le masculin est imposé. Il s'avère que les filles sont nombreuses lorsque les personnages conducteurs sont de sexe féminin et qu'au contraire leur poids est réduit en l'absence de conductrices (graphique 2). Un tel constat ne se vérifie pas chez les garçons.

La proportion des enfants endossant une fonction pédagogique de substitut de l'élève est de $37 \%$; elle varie entre $25 \%$ pour la collection B et environ $40 \%$ pour les autres collections ( $44 \%$ en A et $40 \%$ en C). Les filles ne sont pas discriminées dans l'accès à cette fonction pédagogique. Les proportions de substituts chez les filles et chez les garçons sont équivalentes au sein des collections A ( $42 \%$ des garçons et $46 \%$ des filles sont des substituts) et $B$ ( $26 \%$ des garçons et $24 \%$ des filles sont des substituts). De ce fait là encore, les personnages de filles se maintiennent sur le devant de la scène. Dans la collection $C$, la fonction de substitut est nettement plus présente chez les garçons : $47 \%$ des garçons ont une fonction de substitut versus $28 \%$ chez les filles.

Ainsi ces fonctions particulières que peuvent endosser les personnages sont des instruments de la parité, qui permettent aux illustrateurs et illustratrices de penser aux filles et de les inclure systématiquement. 
Graphique 2 : Part des personnages enfants conducteurs et des autres enfants selon le sexe et la collection (en \%)

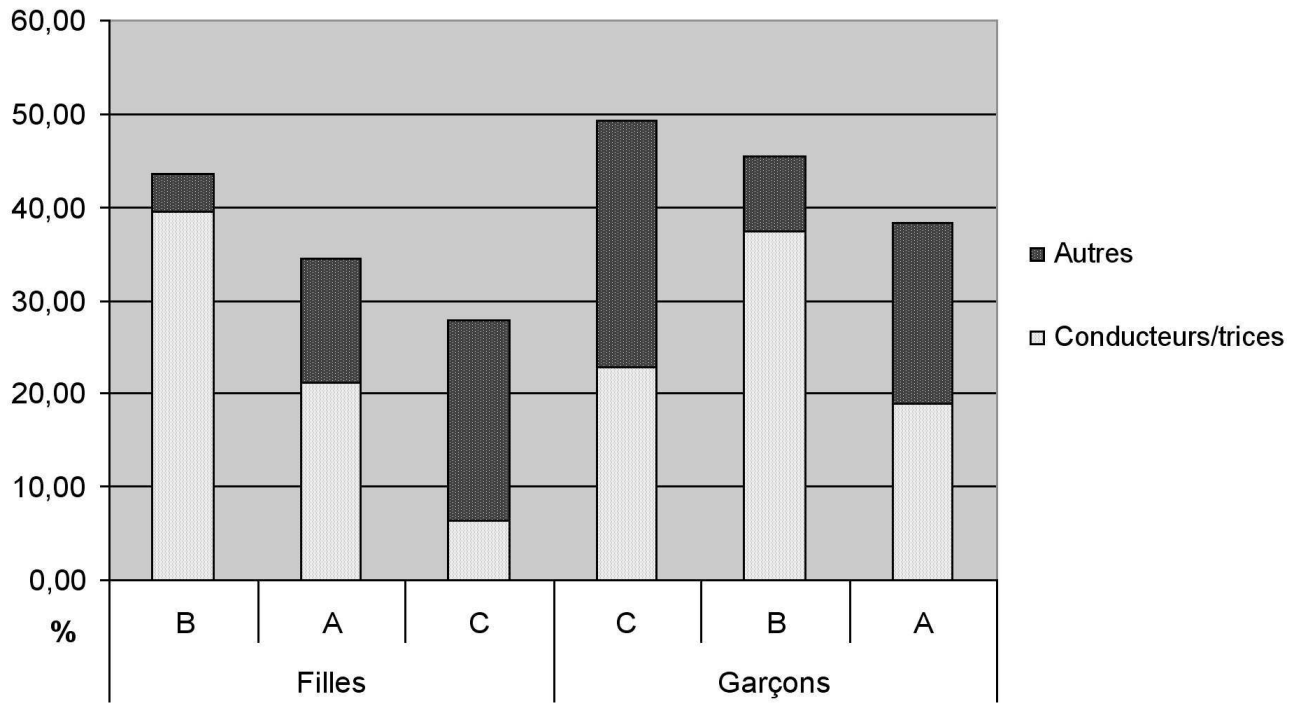

Source : Genre et manuels scolaires en France.

Lecture : Dans la collection B, 44 \% des personnages sont des filles et $40 \%$ sont des fillesconductrices.

La part importante des substituts - représentant un-e élève ou un-e maitresse - et la prégnance des représentations de l'environnement scolaire au sein du manuel expliquent que les activités scolaires priment largement, pour les personnages des deux sexes: les proportions d'enfants réalisant un travail scolaire oscillent entre $39 \%$ et $53 \%$, chez les filles comme chez les garçons. Si on peut relever de légères différences selon les collections, en faveur de l'un ou l'autre sexe, globalement le statut d'écolier-e «neutralise » les représentations des enfants, d'autant que filles et garçons sont souvent présentés sur la même image.

Au vu de ces différences mineures, grâce à la suprématie de la figure de l'écolier-e, seraitce à dire qu'il n'y a pas de construction de la différence des sexes, voire d'inégalité entre filles et garçons?

Paradoxalement, les attributs scolaires, qui en toute cohérence constituent l'attribut majeur des enfants, sont davantage l'apanage des garçons. Dans deux collections au moins, les filles effectuent plus fréquemment une activité scolaire, tout en ayant un moindre accès au matériel scolaire. Outre les activités scolaires, les enfants s'adonnent à des activités ludiques et domestiques. Or, un peu plus les garçons que les filles ont des pratiques de jeu et de loisir, tandis que les activités domestiques, très rares il est vrai, sont, légèrement plus souvent effectuées par les filles qui disposent d'un peu plus d'attributs domestiques, de petits objets faisant parfois écho à la vie quotidienne.

Ces légers écarts dans les activités et les attributs s'accompagnent de dissemblances dans la posture: les garçons sont plus souvent montrés en action, crayon ou matériel de géométrie en main, parfois avec à leurs côtés une fille bras ballants, qui regarde, indique, commente...

De manière classique, l'empreinte du genre est activée dans l'aspect physique du personnage. Les filles ont le plus souvent une chevelure longue ou mi-longue ou bien des nœuds dans les cheveux : c'est le cas des trois quarts des filles. Par exemple, pour citer les 
conductrices que l'élève va suivre tout au long de l'année, dans J'apprends les maths, les deux conductrices, Perrine au $\mathrm{CP}$ et Mathilde aux autres niveaux, portent des cheveux longs; il en est de même pour Léa, conductrice en CP et CE1 de Pour comprendre les mathématiques et de Zoé, Lisa, Maïa de Cap math, conductrices au CP, CE1 et CE2. La jupe ou la robe, vêtements traditionnellement féminins, ne sont pas absentes des images et entrent en concurrence avec le port du pantalon, quand les filles sont vues en pied (ce qui n'est certes pas, très fréquent, les personnages apparaissant le plus souvent en buste). C'est d'ailleurs souvent en cas de courtes chevelures que jupe ou robe font leur apparition.

Outre la chevelure, l'approche qualitative révèle la persistance de couleurs dichotomiques pour distinguer les deux sexes, le rose et le bleu (ou le vert). Mathéo auquel nous avons fait allusion est vert; Mathieu et Mathilde (une blonde à queue de cheval), les deux personnages conducteurs de CM2 de J'apprends les maths, sont respectivement en bleu et en rose.

16 Les couvertures, pourtant "produit d'appel » et objet d'un soin particulier des maisons d'édition, n'échappent pas aux stéréotypes que nous venons d'énoncer, en termes de coiffure, de couleur ou de posture. La couverture de Pour comprendre les mathématiques de CE1 montre en gros plan un couple d'enfants : la fille en jupe est dessinée de dos, tenant règle et équerre, alors que le garçon est de face et se livre à l'élaboration de figures géométriques ${ }^{3}$ (celle de CE2 est construite sur le même schéma) ${ }^{4}$. Et quand Cap math montre une fille comme le garçon en tenue de cosmonaute, la fille est dotée de couettes tenues par élastiques roses.

La construction du genre qui s'amorce chez les enfants s'affirme chez les adultes.

\section{Un monde adulte minoré mais fortement genré}

Les adultes sont minoritaires dans les images, ne représentant globalement que $21 \%$ des personnages. Les hommes sont, de manière écrasante, plus nombreux (564 hommes contre 196 femmes, soit $74 \%$ d'hommes et $26 \%$ de femmes). Les adultes mènent deux types d'activités qui définissent deux sphères de vie: les activités professionnelles, qui sont largement prépondérantes, inscrivent les personnages dans la sphère publique, tandis que les activités extra-professionnelles délimitent une sphère privée.

Pour les hommes comme pour les femmes, l'occupation majeure est l'activité professionnelle. Mais, alors que plus de $60 \%$ des hommes sont des actifs, seules $42 \%$ des femmes le sont. Seuls les hommes étendent leur champ d'action dans toute la sphère publique en accédant aux dix secteurs professionnels sur les dix répertoriés dans la société fictive des manuels (graphique 3). En dehors de l'enseignement, ils l'emportent numériquement dans tous les métiers. Les femmes ont accès à la sphère publique de manière restrictive: elles sont bannies de 4 secteurs, les milieux artistiques ou intellectuels, les métiers d'ordre ou de sécurité, l'artisanat ou les travaux publics et... l'aventure. À cet égard, la couverture de Pour comprendre les mathématiques de CM2 est explicite: au premier plan, une scène de rue peuplée d'ouvriers (des hommes exclusivement) sur un chantier, et une femme avec un enfant ; en arrière-plan, une salle de classe avec une maitresse et une fille au tableau, toutes deux en jupe et cheveux longs ${ }^{5}$. Grâce notamment à la fonction de substitut, les femmes sont hégémoniques dans le secteur de l'éducation. Mais l'image de l'enseignant varie selon le sexe. Les femmes légèrement plus nombreuses sont moins dotées en matériel et ne sont jamais dépeintes, 
contrairement à certains enseignants, comme des scientifiques ou des savantes. On pense par exemple au personnage récurrent de Monsieur cuBus dans J'apprends les maths, caricature de savant avec la tête au carré, des cheveux blancs et le crâne dégarni, affublé de lunettes. Quant aux métiers de commerce, (la plupart du temps alimentaires), et de service (où elles exercent), ils rappellent, tout comme l'éducation d'ailleurs, l'assignation des femmes à la sphère privée et les tâches qui leur sont traditionnellement dévolues.

Graphique 3 : Répartition des personnages adultes selon leur sexe par secteur professionnel (en effectifs)

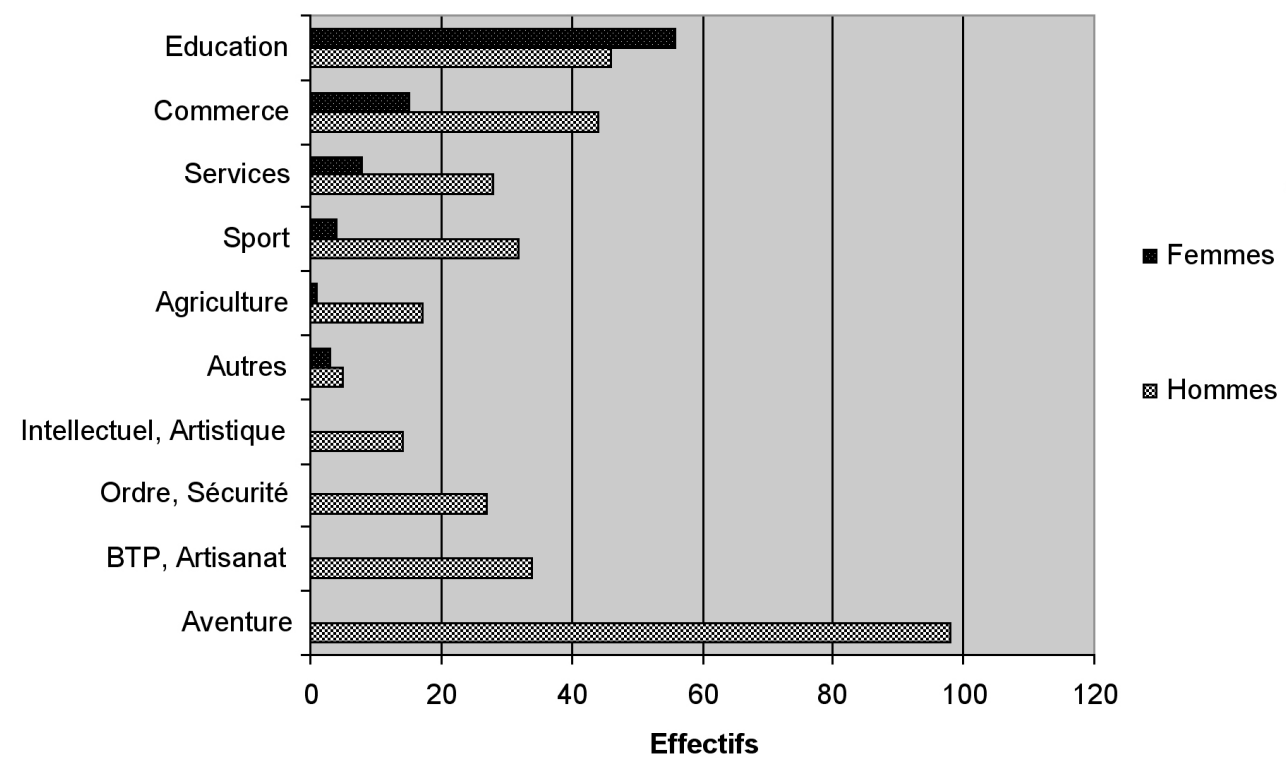

Source : Genre et manuels scolaires en France

Les activités extra-professionnelles couvrent trois grands domaines: les loisirs (sport, culture...), les activités domestiques (ménage, cuisine, achat...), les activités de la vie quotidienne pour soi (manger, dormir, se déplacer...). Elles sont toutes, comme le champ professionnel, davantage investies par les hommes, puisqu'ils sont plus nombreux (graphique 4). En outre, hommes et femmes n'occupent pas leur temps extraprofessionnel de façon identique. Les hommes privilégient nettement les loisirs, puis se partagent entre activités domestiques et activités de la vie quotidienne. Chez les femmes, loisirs et tâches domestiques se concurrencent tandis que les activités de la vie quotidienne restent en retrait.

En résumé, les hommes par leurs activités s'imposent dans le champ social. Les femmes, qui se déploient dans les deux sphères, sont cantonnées, autant dans le privé que dans le public, dans des activités traditionnellement féminines. Les élèves peuvent en retenir l'implication ménagère préférentielle des personnages féminins. 
Graphique 4 : Répartition des personnages adultes selon leur sexe par domaine d'activité extraprofessionnelle (en effectifs)

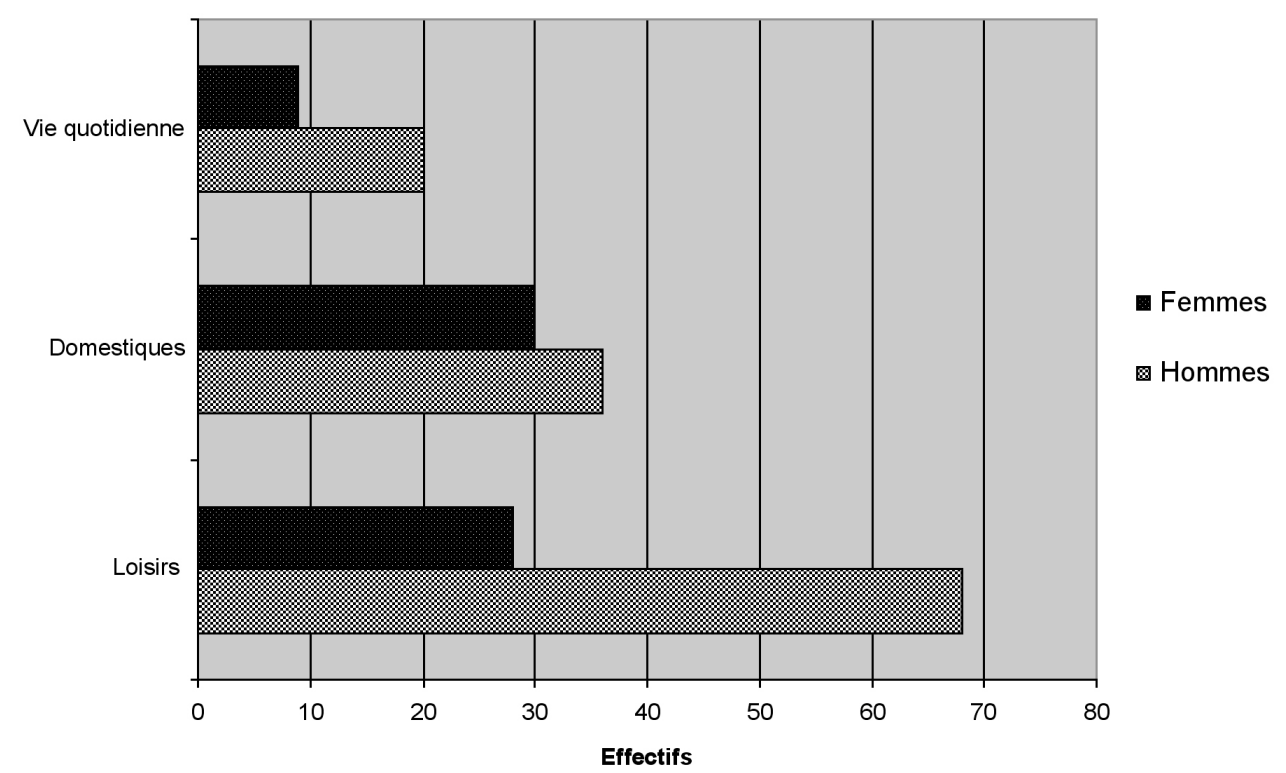

Source : Genre et manuels scolaires en France

\section{En guise de conclusion}

Avec le nombre important d'enfants et l'omniprésence de l'univers scolaire, les manuels en France témoignent de la place majeure de l'enfant et de la scolarisation dans la société française. Dans nos manuels d'aujourd'hui, se lit aussi la sensibilité au "discours mythique » de l'égalité, selon les termes de Llana Löwy (2006), et aux pressions sociales de lutte contre les discriminations, du moins à l'égard du sexe féminin, car la pluralité des origines et des cultures reste quant à elle peu visible. À la bipolarisation et la hiérarchisation entre les sexes s'est substitué un système de genre reposant sur l'opposition de deux sphères : d'une part celle de l'enfance aux similitudes numériques et qualitatives entre garçons et filles, d'autre part celle de l'âge adulte où les femmes constituent une minorité, aux marges de manœuvre limitées, malgré le refus de l'exclusion. Ainsi, les images des manuels traduisent la tension entre un contrôle manifeste du sexisme et la croyance en une nécessaire différence des sexes dont il faudrait, malgré tout, marquer la distinction.

\section{BIBLIOGRAPHIE}

BAUDELOT C., ESTABLET R., Quoi de neuf chez les filles ? Entre stéréotypes et libertés. Paris : Nathan.

L'enfance en questions, 2007 
BAUDINO C., Revue de littérature récente sur les inégalités de genre dans les méthodes d'enseignement et la gestion des relations entre pairs dans l'aire francophone. Document de référence préparé pour le rapport mondial de suivi sur l'éducation pour tous de 2008. Paris : Unesco, 2007

Boisseau A., Chauveau V., Delon F., MADEC G., Du côté des mathématiciennes. Lyon : Aléas, 2002

BLUMBERG R. L., Gender bias in textbooks : a hidden obstacle on the road to gender equality in education. Background paper for the Education for all global monitoring report 2008 : Education for all by 2015 : will we make it? Unesco : 2008/ED/EFA/MRT/PI/18, 2007

BRAUN A., «Les manuels... du grain à moudre pour la recherche en éducation ». Revue Éducation et formation. Manuels scolaires et matériels didactiques, ne-292, janvier, Université de Mons, 2010 BRUGEILLES C., CROMER S., Analyser les représentations du masculin et du féminin dans les manuels scolaires. Paris : CEPED. Collection Les Clefs Pour (téléchargeable sur http://www.ceped.org/IMG/ pdf/les_clefs_pour_brugeilles.pdf), 2005

BRUGEILLES C., CROMER S., " Les manuels scolaires de mathématiques ne sont pas neutres. Le système de genre d'une collection panafricaine de l'enseignement primaire ", Autrepart, n³9, p. 149-166, 2006

BRUGEILLES C., CROMER S., Représentations sexuées dans des manuels de mathématiques d'enseignement primaire en Afrique. In Du genre et de l'Afrique. Hommage à Thérèse LocoH (coord. J. VALLIN), Ined, p.83 à 95, 2009

BRUGEILLES C., CROMER S., Promouvoir l'égalité entre les sexes par les manuels scolaires. Un guide pour les acteurs et actrices de la chaîne du livre. Paris : Unesco (téléchargeable sur unesco.doc), 2008

BRUILLARD E., Les manuels scolaires questionnés par la recherche, in BRUILLARD E. (dir.), Manuels scolaires, regards croisés, CRDP de Basse Normandie, documents, actes et rapports de l'Éducation, p. 13-36, 2005

DURPAIRE J.-L., L'enseignement des mathématiques au cycle 3 de l'école primaire. Rapport IGEN 2006-034, 2006

EVANS L., DAVIES, K., « No Sissy Boys Here : A content analysis of the representation of masculinity in elementary school reading textbooks ». Sex roles : A journal of research, Vol. 42, N 3-4 : 255-70, 2000

JARLEGAN A. (Coord.), Dossier Genre et éducation, institutions, pratiques, représentations, Recherches et éducations $\mathrm{n}^{\circ} 2$, Société BINET-SIMON, 2009

JODELET D., Les représentations sociales. Paris : PUF, 1989

JACKSON S., GEE S., « Look Janet », « No you look John » : constructions of gender in early school reader illustrations across 50 years'. Gender and education, 17 (2) : 115-128, 2005

LöWY L., L'emprise du genre : masculinité, féminité, inégalité. Paris : La Dispute, Coll. Le genre du monde, 2006

MICHEL P., « Concours de recrutement de professeurs des écoles Session 2004 ». Note d'information 05-17. MEN-D.E.P., mai 2005

POMART C., Analyse des représentations du féminin et du masculin dans les manuels de sciences, de collège, utilisés en 2008-2009 à Toulouse. Mémoire de Master 1 Université Toulouse II Le Mirail, 98 p. 2009

Réseau Eurydice « Éducation, audiovisuel et culture », Différences entre les genres en matière de réussite scolaire: étude sur les mesures prises et la situation actuelle en Europe. (http:// www.eurydice.org), 2010 
TISSERANT P., WAGNER A.-L. (dir.), Place des stéréotypes et discriminations dans les manuels scolaires. Rapport pour la HALDE, 2008

UNESCO, Genre et éducation pour tous : le pari de l'égalité. Éducation pour tous (EPT), Rapport mondial de suivi. Unesco, 2003-2004

\section{Corpus analysé}

Cap Math, CP au CM2, Paris : HATIER

CP : CHARNAY R., DUSSUC M-P., MADIER D., 2005

CE1 : CHARNAY R., DUSSUC M-P., MADIER D., 2006

CE2 : CHARNAY R., COMBIER G., DUSSUC M-P., MADIER D., 2007

CM1 : COMBIER G., CHARNAY R., DUSSUC M-P., 2008

CM2 : COMBIER G., CHARNAY R., DUSSUC M-P., 2008

J'apprends les maths, $\mathrm{CP}$ au CM2, Paris : RETZ

CP : sous la direction de BRISSIAUd R., LELIÈVRE F., CLERC P., OUZOULIAS A., SUIRE F., 2008

CE1 : BRISSIAUD R., CLERC P., OUZOULIAS, A., 2002

CE2 : BRISSIAUD R., CLERC P., LELIÈVRE F., OUZOULIAS A, 2003

CM1 : BRISSIAUD R., CLERC P., LELIÈVRE F., OUZOULIAS A, 2005

CM2 : BRISSIAUD R., CLERC P., LELIÈVRE F., OUZOULIAS A, 2006

Pour comprendre les mathématiques, $\mathrm{CP}$ au CM2, Paris : HACHETTE

CP : BLANC, J-P., BRAMAND P., DEBÛ P., DUbOIS A., LAFONT E., PEYNICHOU D., TRUANT D., VARGAS A., 2008

CE1 : BLANC J-P., BRAMAND P., DEBÛ P., GÉLY J., PEYNICHOU D., VARGAS A., 2004

CE2 : BLANC J-P., BRAMAND P., DEBÛ P., GÉLY J., PEYNICHOU D., VARGAS A., 2004

CM1 : BLANC J-P., DUBoIS A., BRAMAND P., DEBÛ P., GÉLY J., PEYNICHOU D., VARGAS A., 2007

CM2 : BLANC J-P., BRAMAND P., DEBÛ P., GÉLY J., LAFONT E., PEYNICHOU D., VARGAS A., 2005

\section{NOTES}

1. L'égalité entre les sexes dans l'éducation, en éliminant les disparités dans les enseignements primaire et secondaire à échéance 2015 est un objectif de l'Éducation pour tous (EPT, Dakar, 2000, objectif 5 ; cf. Unesco) et du Millénaire pour le Développement (objectif 3 ; cf. Programme des Nations Unies pour le développement).

2. Pour une présentation détaillée de la méthode: BRUGEILLES, CROMER, 2005 et pour une méthodologie simplifiée, BRUGEILLES, CROMER, 2008. Ces deux ouvrages sont téléchargeables sur internet et disponibles aussi en anglais.

3. Dans l'édition 2009, la couverture a changé : au premier plan, le garçon blond saute sur un gros ballon, au second plan une fille rousse à couette en pantalon et lunettes roses semble courir et en arrière-plan un second garçon jouant au cerf-volant.

4. En revanche, sur la couverture du CP de l'édition 2003, c'est le garçon qui est de dos. 
5. L'image est reprise dans le corps du manuel avec un cadre élargi, qui ajoute : un musicien, un dessinateur, une homme se rendant au travail (avec sa mallette), une vieille femme...

\section{RÉSUMÉS}

Cet article étudie les représentations de genre circulant dans les illustrations de trois collections de manuels scolaires de mathématiques parmi les plus diffusées en France en 2008. Persistent la dominance numérique des personnages masculins et, pour les adultes, des portraits différenciés selon le sexe, mis en évidence dans d'autres études. Cependant, une tendance à la parité et à l'égalité se dessine chez les enfants, personnages majoritaires. Le contrôle du sexisme est assuré par l'utilisation de personnages récurrents des deux sexes qui guident les élèves tout au long de leurs apprentissages.

This article studies gender representations, as portrayed in pictures of three series of mathematics textbooks, the series under study being the most popular in France in 2008. The presence of more male figures and the prevalence of stereotypical portraits according to the sex of the adult depicted still confirm what previous studies had already shown. However a trend towards equality in numbers and equity in sex roles appears when children are depicted, which represent the majority of the cases. Sexism is controlled thanks to recurrent characters of both sexes who are guiding the pupils throughout the time of their learning.

\section{INDEX}

Mots-clés : genre, manuel scolaire, mathématiques, représentations sexuées

Keywords : gender, gender representations, mathematics, textbook

\section{AUTEURS}

\section{CAROLE BRUGEILLES}

Université Paris Ouest Nanterre, CERPOS, France

\section{SYLVIE CROMER}

Université Lille 2, Droits et perspectives du droit, Université Lille Nord de France-UDSL, France 